\title{
The Role of The RNA Demethylase FTO (Fat Mass and Obesity-Associated) and mRNA Methylation in Hippocampal Memory Formation
}

\author{
Brandon J Walters ${ }^{1,2,3}$, Valentina Mercaldo $1,2,3$, Colleen J Gillon ${ }^{1,2}$, Matthew Yip ${ }^{1,2,3}$, Rachael L Neve ${ }^{4}$, \\ Frederick M Boyce ${ }^{5}$, Paul W Frankland ${ }^{1,2,3}$ and Sheena A Josselyn*, 1,2,3 \\ 'The Hospital for Sick Children, Department of Neuroscience and Mental Health, Toronto, Ontario, Canada; ${ }^{2}$ University of Toronto, Department of \\ Physiology, Toronto, Ontario, Canada; ${ }^{3}$ University of Toronto, Department of Psychology, Toronto, Ontario, Canada; ${ }^{4}$ Massachusetts Institute of \\ Technology, Department of Brain and Cognitive Sciences, Cambridge, MA, USA; ${ }^{5}$ Massachusetts General Hospital, Department of Neurology, \\ Cambridge, MA, USA
}

\begin{abstract}
The formation of long-lasting memories requires coordinated changes in gene expression and protein synthesis. Although many studies implicate DNA modifications (DNA methylation, histone modifications) in memory formation, the contributions of RNA modifications remain largely unexplored. Here we investigated the role of mRNA methylation in hippocampal-dependent memory formation in mice. RNA modifications are highly dynamic and readily reversible. Methyltransferases add a methyl group to mRNA while demethylases remove methyl groups. Here we focused on examining the role of the best characterized RNA demethylase, FTO (fat mass and obesityassociated) in memory. We observed that FTO is expressed in the nuclei, dendrites and near dendritic spines of mouse dorsal hippocampal CAI neurons. Next, we found that contextual fear conditioning transiently $(0.5 \mathrm{~h})$ decreased Fto levels in these neurons, with the largest decrease in FTO observed near synapses. The decrease in FTO observed shortly after contextual fear conditioning suggests that FTO normally constrains memory formation. To directly test this, we artificially decreased FTO levels in dorsal hippocampus of otherwise normal (wild-type) mice by microinjecting before training a single herpes simplex virus (HSV) vector expressing either CRISPR/Cas9 or shRNA targeted against Fto. Decreasing FTO using either method specifically enhanced contextual fear memory. Together, these results show the importance of FTO during memory formation and, furthermore, implicate mRNA modification and epi-transcriptomics as novel regulators of memory formation.

Neuropsychopharmacology (2017) 42, I502-1510; doi:I0.1038/npp.2017.31; published online I5 March 2017
\end{abstract}

\section{INTRODUCTION}

The formation of long-lasting memories requires coordinated changes in gene expression and protein synthesis (Davis and Squire, 1984; Duvarci et al, 2008; Hernandez and Abel, 2008). Previous research shows the important roles of histones and DNA modifications (epigenetics) in memory formation (Sweatt, 2013; Zovkic et al, 2014). However, the potential role of RNA modifications (epi-transcriptomics) in memory formation has only begun to be explored. RNA can be subjected to over 100 modifications, each of which may produce distinct functional consequences (Fu et al, 2014; Hoernes et al, 2015; Jia et al, 2013). The most abundant RNA modification is methylation of adenosine bases at the

* Correspondence: Dr SA Josselyn, Department of Psychology, Physiology, IMS, Hospital for Sick Children, University of Toronto, 555 University Avenue, Toronto, Ontario M5G IX8, Canada, Tel: + I 416 8I3 7654 ext 301824, Fax: + 4 416 813 7717,

E-mail: Sheena.Josselyn@SickKids.ca

Received I December 2016; revised 13 January 2017; accepted 2 February 2017; accepted article preview online 16 February 2017 nitrogen-6 position (referred to as m6A; Liu and Pan, 2016; Niu et al, 2013). The precise function of the m6A modification is unknown, but several lines of evidence suggest this modification regulates many structural and functional properties of mRNA, including translation (Dominissini et al, 2012; Hoernes et al, 2015; Linder et al, 2015; Schwartz et al, 2014; Wang et al, 2015; Zhao et al, 2014; Zhou et al, 2015). Precise mRNA translation is necessary for memory formation, thereby implicating the $\mathrm{m} 6 \mathrm{~A}$ modification of mRNA in the regulation of memory formation. Therefore, epi-transcriptomics, specifically the m6A modification of mRNA, may represent a novel mediator of neural plasticity and memory formation.

The m6A modification of mRNA is highly dynamic and readily reversible (Jia et al, 2013). Methyltransferases ('writers' of methylation) add the methyl group to mRNA while demethylases ('erasers' of methylation) are responsible for removing this modification. Fat mass and obesityassociated protein (FTO) is the best characterized demethylase. FTO is highly enriched in the brain compared to muscle (McTaggart et al, 2011) and previous evidence shows that 
FTO is involved in neural dopaminergic signaling (Hess et al, 2013). In vitro studies find that demethylation of RNA by FTO is stimulus-dependent (Zhou et al, 2015), suggesting the m6A modification may be important in experience dependent plasticity. Consistent with this notion, a recent study implicated the m6A modification of mRNA in the medial prefrontal cortex in auditory cue fear memory formation in mice (Widagdo et al, 2016). However, the m6A modification is enriched throughout the brain (Biogps. org, (Meyer et al, 2012)) suggesting this modification may also play a role in memory formation in other brain regions. Here we use a combination of behavioral studies, molecular biology, and a novel CRISPR/Cas9 virus to examine whether regulation of mRNA methylation by FTO in the dorsal hippocampus, a key region for memory formation (Frankland et al, 1998; Sanders et al, 2003; Sekeres et al, 2010; Wiltgen et al, 2006; Wiltgen et al, 2010), plays a role in learning and memory.

\section{MATERIALS AND METHODS}

\section{Mice}

Adult (10-12 weeks old) male F1 hybrid (C57 BL/6NTac $\times$ 129S6/SvEvTac) naive wild-type (WT) mice were used for all experiments. Mice were group housed (2-5 mice per cage) on a $12 \mathrm{~h}$ light/dark cycle and provided with food and water ad libitum. All experimental procedures were conducted in accordance with the guidelines of the Canadian Council on Animal Care (CCAC), National Institutes of Health (NIH) and approved by the Animal Care Committee at the Hospital for Sick Children.

\section{Stereotaxic Surgery}

Mice were pretreated with atropine sulfate $(0.1 \mathrm{mg} / \mathrm{kg}$, i.p.), anesthetized with chloral hydrate $(400 \mathrm{mg} / \mathrm{kg}$, i.p.) and placed in a stereotactic frame. The scalp was incised and bilateral skull holes drilled. Viral vectors were bilaterally microinjected $(2.0 \mu \mathrm{l}$ per side, $0.1 \mu \mathrm{l} / \mathrm{min})$ into the CA1 region of the dorsal hippocampus (co-ordinates, -2.3, $\pm 1.5-1.5 \mathrm{~mm}$ relative to bregma (Paxinos and Franklin, 2004)). Micropipettes remained in place for $10 \mathrm{~min}$ after microinjection to ensure vector diffusion. Micropipettes were slowly withdrawn, the incision closed and mice treated with analgesic (ketoprophen, $5 \mathrm{mg} / \mathrm{kg}$, s.c.).

\section{Viral Constructs}

CRISPR/Cas9. sgRNA against Fto was designed using Desktop Genetics (www.deskgen.com, 5'-GCAGTGTGAGAAAGGCCTC-3'). sgRNA-Fto was validated in-house before being subcloned into an all-in-one CRISPR system for use with HSV at the Viral Vector Core at McGovern Institute (MIT). The basic cassette is as follows: hSyn-Cas9WPRE-U6-gRNA scaffold. Cas9-Control was made using a similar procedure.

shRNA/scrambled shRNA. Pre-validated shRNA-Fto was obtained from Sigma-Aldrich (TRCN0000277193, sequence 5'- GTCTCGTTGAAATCCTTTGAT-3') and scrambled shRNA (shRNA-scramble) was a gift from David Sabatini
(Sarbassov et al, 2005; Addgene \#1864). Both shRNA-Fto and shRNA-scramble were sub-cloned into a duel expression HSV vector with CMV driving GFP expression and U6 driving expression of shRNA.

Replication-deficient HSV-derived particles were made from these vectors as previously described (Han et al, 2009). The titer of these HSV was $>1 \times 10^{8}$ infectious units $/ \mathrm{ml}$. We chose to use HSV to deliver our constructs of interest because HSV is naturally neurotropic (Cole et al, 2012; Steiner et al, 2007) and has large packaging limits, allowing exceptionally large constructs, such as Cas9, to be accommodated (Saeki et al, 2001). This is crucial for our CRISP/ Cas9 approach, as other viruses (including AAV) cannot accommodate the necessary transgenes to allow single-vector manipulations in WT mice.

\section{Behavioral Training}

Mice were handled each day for 3 days prior to contextual fear conditioning. During contextual fear conditioning, mice were placed in a fear conditioning chamber for $2 \mathrm{~min}$, then three unsignalled $0.5 \mathrm{~mA}$ foot shocks spaced $1 \mathrm{~min}$ apart were delivered. Mice remained in the fear conditioning context for 1 additional min before being returned to the homecage.

For experiments characterizing the dynamics of Fto expression (Figure 3), mice were kept in a holding area until their brains were flash-frozen 0.5 or $1 \mathrm{~h}$ after training. Hippocampi were dissected from the whole brain followed by isolation of area CA1 of the dorsal hippocampus.

For knockdown experiments (Figure 4) a weaker training protocol was used $(1 \times 0.5 \mathrm{~mA}$ foot shock) to allow potential increases in memory to be observed. To test memory, mice were returned to the same context $24 \mathrm{~h}$ after training. The percentage of time mice spent freezing (defined as adoption of an immobilized, crouched position, with an absence of any movement except respiration (Blanchard and Blanchard, 1969; Bolles and Fanselow, 1982) during the 5 min test was recorded (Freezeframe software; Actimetrics). Mice were then either perfused (transcardially with $0.1 \mathrm{M}$ PBS followed by $4 \%$ PFA) for immunohistochemistry or the dorsal CA1 region of the hippocampus was harvested for qPCR.

To examine the reactivity of mice to the foot shock, video recordings of mouse behavior during the training session were imported into Ctrax (http://ctrax.sourceforge.net/, (Branson et al, 2009)). The velocity of each mouse during baseline (measured over 120 s) and foot shock (measured during the $2 \mathrm{~s}$ foot shock) were compared as an indicator of pain reactivity (Anagnostaras et al, 2000). This value was normalized to the baseline and experimental groups were compared to their respective control groups (HSV-Cas9Control or HSV-shRNA-Scramble). Post-shock freezing was measured for $60 \mathrm{~s}$ after delivery of the final shock as a measure of within-session learning (Anagnostaras et al, 2000; Wood and Anagnostaras, 2011).

\section{RNA Isolation/cDNA Synthesis}

RNA from the CA1 region of dorsal hippocampus or Neuro2A (N2a) cells was isolated using a kit (EZ-10 RNA isolation kit, Bio-Basic, BS82322). For mRNA enrichment (Figure $3 \mathrm{~d}$ ), $26 \mu \mathrm{l}$ of the $30 \mu \mathrm{l}$ of isolated RNA was further 
purified (MagJet mRNA enrichment kit, ThermoFisher, K2811) and concentrated to $14 \mu \mathrm{l}$ (RNEasy MinElute cleanup kit, Qiagen, 74204). Total RNA was quantified using a Nanodrop 1000 (ThermoFisher). For gene enrichment studies or mRNA normalization (Figure 3d), RNA was converted into cDNA (400 ng for gene enrichment; $2 \mu \mathrm{l}$ mRNA for mRNA normalizations) using a kit (High Capacity cDNA synthesis kit, ThermoFisher, 4368814).

\section{Quantitative Real-Time PCR (qPCR)}

qPCR was performed on $2 \mathrm{ng}$ of cDNA in a $10 \mu \mathrm{l}$ reaction (EvaGreen Mastermix, Diamed, ABM Mastermix-S). To normalize m6A-containing mRNA (Figure 3d), we loaded $2 \mu \mathrm{l}$ of cDNA in a standard $10 \mu \mathrm{l}$ PCR reaction. Relative enrichment was calculated using the $\Delta \Delta C T$ method, using the geometric mean of two internal controls (Hprt, Gapdh) for normalization. qPCR was run on a CFX96 real-time qPCR detection system (BioRad) using $500 \mathrm{nM}$ primer dilutions. All qPCR reactions were run in triplicates.

Primers $\left(5^{\prime}-3^{\prime}\right.$, forward-reverse)

Alkbh5: (5'-GGCGTTCCTTAATGTCCTGA-3', 5'-AGTT CCAGTTCAAGCCCATC-3')

Fto: (5'-CTCAGCCACTCAAACTCCAC-3', 5' -TCTTAGA ACGCTGTCAGTTGG-3')

Gapdh: (5'GTGGAGTCATACTGGAACATGTAG-3', 5'-AA TGGTGAAGGTCGGTGTG-3')

Hprt: (5'-GGAGTCCTGTTGATGTTGCCAGTA-3', 5'-G GGACGCAGCAACTGACATTTCTA-3')

\section{m6A ELISA}

m6A-containing RNA was quantified using EpiQuik RNA methylation quantification kit (Epigentek, P-9005). Either $200 \mathrm{ng}$ of total RNA or $8 \mu \mathrm{l}$ of concentrated mRNA were run and fit to a standard curve. Total RNA samples were then normalized against the total amount (ng) of RNA loaded, while mRNA samples were normalized to the geometric mean of two internal controls (Hprt, Gapdh) determined from qPCR, as the concentration of mRNA was below the detection limit of the Nanodrop 1000. All samples were run in triplicate.

\section{FTO, GFAP, GAD67, Parvalbumin and m6A Immunostaining in Brain Slices}

Coronal brain sections $(50 \mu \mathrm{m})$ from homecage WT mice were incubated with blocking solution $(0.1 \%$ BSA, $2 \%$ NGS, $0.3 \%$ Triton-X) for $2 \mathrm{~h}$ at RT, followed by incubation with rabbit anti-FTO polyclonal (1 : 100; Novusbio, NB110-60935) and one of the following primary antibodies: mouse monoclonal anti-GFAP (1:500; Cell Signaling Technology), mouse monoclonal anti-GAD67 (1:500; Millipore) or mouse monoclonal anti-parvalbumin (1:500; Sigma-Aldrich) at RT for $48 \mathrm{~h}$. Sections were washed and then incubated with goat-anti-rabbit/mouse Alexa 568/633 (1:500; Invitrogen) for $2 \mathrm{~h}$ at RT.

Supplementary Figure S1A. Brain sections $(50 \mu \mathrm{m})$ from naive mice were incubated with blocking solution as above and then incubated with rabbit anti-FTO monoclonal (1 : 100; gift from Dr Uli Rüther, (Hess et al, 2013) at RT for $48 \mathrm{~h}$. Sections were washed and then incubated with goatanti-rabbit Alexa 488 (1:500; Invitrogen) for $2 \mathrm{~h}$ at RT.

Supplementary Figure S1B. Brain sections $(50 \mu \mathrm{m})$ from naive mice were incubated with blocking solution as above and then incubated with rabbit anti-m6A (1:300; Abcam) primary antibody at RT for $48 \mathrm{~h}$. Sections were washed and then incubated with goat-anti-rabbit Alexa 488/568 (1:500; Invitrogen) for $2 \mathrm{~h}$ at $\mathrm{RT}$.

\section{FTO and m6A Immunostaining in Primary Hippocampal Neurons}

Primary hippocampal neurons were prepared as described in (Cole et al, 2012). After 10d in culture, neurons were washed, fixed with $4 \%$ paraformaldehyde in PBS, permeabilized and blocked with $0.3 \%$ Triton $\mathrm{X}-100,2 \%$ normal goat serum and $0.5 \%$ bovine serum albumin in PBS. Neurons were incubated at RT for $2-3 \mathrm{~h}$ with mouse anti-FTO (1:100, Novus Biologicals, NB110-60935, 1:100) and anti-m6A (1:300; Abcam) in blocking solution. After washes, neurons were incubated with goat anti-mouse/rabbit Alexa 488/568 secondary antibodies (1:500, Invitrogen) in blocking solution for $1 \mathrm{~h}$ at $\mathrm{RT}$, washed and mounted.

\section{FTO and GFP Staining for Spine Detection}

To examine FTO expression within dendritic spines, we took advantage of an HSV viral vector expressing GFP throughout infected neurons. Homecage mice were microinjected with HSV-GFP into the CA1 region of dorsal hippocampus. Infected neurons were filled with GFP, allowing morphological identification of spines. Five days later, mice were perfused. Brain sections $(50 \mu \mathrm{m})$ were incubated with blocking solution as above and then incubated with mouse anti-FTO monoclonal (Novus Biologicals) and anti-rabbit GFP (1:500; Invitrogen, AB290) at RT for $48 \mathrm{~h}$. Sections were washed, then incubated with goat-anti-rabbit/mouse Alexa 488/568 (1:500; Invitrogen) for $2 \mathrm{~h}$ at RT.

For all immunostaining experiments, the sections were mounted with PermaFluor mounting medium (Thermo Scientific) and counterstained with DAPI. Images were acquired using a confocal laser scanning microscope (LSM 710; Zeiss).

\section{Neuro-2A Cell Culture}

N2A cells (ATCC, \#CCL-131) were grown in DMEM modified with $10 \%$ fetal bovine serum, $1 \times$ Glutamax and 1X Penicillin/Streptomycin (all from Gibco). All experiments were performed on N2A cells between passage 6 and passage 10. Cells were transduced at $60 \%$ confluence and gown for $1 \mathrm{~d}$ before fixation ( $4 \%$ fresh cold PFA for $20 \mathrm{~min}$ ). Cells were stained with the indicated antibody following the protocols as above.

\section{Synaptoneurosomal Preparation and Western Blot Analysis}

Synaptoneurosomes were prepared by homogenizing four fresh hippocampi (from two mice) in ice-cold buffer, as previously described (Pilo Boyl et al, 2007). Nuclear and synaptoneurosomal fractions were separated by SDS- 
polyacrylamide gel electrophoresis and electroblotted onto PVDF transfer membranes. Membranes were blocked for $1 \mathrm{~h}$ in 10\% skim milk in TBS and incubated overnight at RT with primary antibodies (FTO, 1:500, Novusbio). Anti-mouse/ rabbit-HRP secondary antibodies were incubated at $4{ }^{\circ} \mathrm{C}$. Optical intensity of bands associated with FTO and $\mathrm{N}$-Cadherin were analyzed using Image $\mathrm{J}$ software. Blots were repeated a minimum of three times.

\section{Statistics}

One-way ANOVAs were used to test for group differences, followed by a Dunnett post hoc analyses on significant main effects. Statistics were performed using the Statistica software package. The Kolmogorov-Smirnov's normality test confirmed that these data were normally distributed.

\section{RESULTS}

\section{Context Fear Conditioning Decreases FTO Levels in Dorsal CA1 Hippocampal Neurons}

Previous studies find that both the demethylase FTO and the m6A modification are enriched in the brain (Dominissini et al, 2012; Meyer et al, 2012). We first examined the expression of FTO specifically within the dorsal hippocampus of mice. Using immunohistochemistry, we found widespread expression of FTO in neurons, interneurons and glial cells (Figure 1). Within neurons, we observed robust staining of FTO in the nucleus and cell body, but we also observed weaker staining outside of the cell body (Figure 1). Using an antibody directed against the m6A modification, we found a similar pattern of staining $\mathrm{m} 6 \mathrm{~A}$ in dorsal hippocampal neurons (the m6A modification was present both inside and outside of neuronal nuclei, Supplementary Figure S1B). Because FTO has not previously been reported in nonnuclear neuronal compartments (McTaggart et al, 2011), we confirmed FTO expression in dendrites in vivo using an additional antibody directed against FTO (Hess et al, 2013; Supplementary Figure S1A) and in isolated hippocampal cultured neurons (Figure 2a). We further verified the presence of FTO in neuronal synapses by preparing synaptoneurosomes (purified synapses containing the preand postsynaptic termini; Figure 2c). Finally, we used HSV expressing GFP to fill the dendrites of infected neurons in vivo and observed FTO staining near morphologically visualized dendritic spines (Figure $2 b$ ). Together, these independent lines of evidence confirm, for the first time, the presence of FTO both within the cell body but also near synaptic spines. The pattern of expression also is consistent with the notion that FTO is important in synaptic plasticity and memory formation.

To examine the role of Fto in memory formation, we first assessed whether levels of Fto, or the other identified RNA demethylase Alkbh5, were affected by contextual fear conditioning. Context fear conditioning is a form of Pavlovian conditioning that critically depends on the CA1 region of dorsal hippocampus (Frankland et al, 1998; Ji and Maren, 2008; Nakazawa et al, 2016; Tanaka et al, 2014). We trained mice and collected dorsal CA1 hippocampal tissue either 0.5 or $1 \mathrm{~h}$ later. Levels of Fto or Alkbh 5 in trained mice were compared to mice in three control groups; (1) experimentally naive mice (taken directly from homecage), (2) mice that received context placement only (no foot shock) either 0.5 or $1 \mathrm{~h}$ before, and (3) mice that received an immediate foot shock upon placement in the context either 0.5 or $1 \mathrm{~h}$ before (a type of training that does not produce strong memory (Fanselow, 1990; Frankland et al, 2004; Wiltgen et al, 2001)). Context fear conditioning transiently decreased Fto expression (Figure 3a). We performed an ANOVA with between-group factor (treatment condition) on the data and found a significant effect $\left(\mathrm{F}_{6,59}=2.55\right.$; $p<0.05)$. Post hoc comparisons revealed that the only group to differ from Homecage control was the Context +Shock group examined $0.5 \mathrm{~h}$ after training. In contrast, contextual fear conditioning did not change expression of Alkbh5, the other identified demethylase $\left(\mathrm{F}_{6,47}=1.47\right.$; $p>0.05$, Figure $3 b$ ).

Given that FTO is a demethylase, our observation that Fto levels are decreased following fear conditioning suggests that the overall level of methylated RNA should be increased after fear conditioning. To test this idea, we similarly trained mice and assessed RNA methylation in both total RNA and purified mRNA obtained from the CA1 region of hippocampus. Contextual fear conditioning did not affect m6A content of total RNA $\left(\mathrm{F}_{2,12}=0.81 ; p>0.05\right.$, Figure $\left.3 \mathrm{c}\right)$, but increased methylated mRNA levels $\left(\mathrm{F}_{2,12}=10.77 ; p<0.05\right.$, Figure $3 \mathrm{~d}$ ), $0.5 \mathrm{~h}$, but not $1 \mathrm{~h}$, after training. Together, these data provide correlational evidence that FTO normally constrains memory formation by decreasing the levels of the m6A modification of mRNA. We next assessed the cellular distribution of FTO within the nuclear and synaptoneurosomal fractions in the hippocampus after context fear conditioning. Compared to homecage controls, we observed a decrease in levels of FTO in the synaptic fraction $0.5 \mathrm{~h}$ after training $\left(\mathrm{F}_{1,10}=12.93 ; p<0.05\right)$, but no change in the nuclear fraction $\left(\mathrm{F}_{1,10}=2.39 ; p>0.05\right.$, Figure 3e). These data are consistent with the interpretation that behavioral training that produces robust memory preferentially reduces FTO levels near synapses.

\section{Artificially Decreasing FTO in Dorsal Hippocampus Enhances Memory}

To directly test whether FTO constrains memory formation by decreasing the m6A modification of mRNA, we artificially decreased FTO levels in WT mice using a single HSV vector to deliver Cas9 targeted against Fto. We chose HSVs because these viral vectors preferentially infect excitatory neurons (Cole et al, 2012) and offer a large packaging capacity (Neve, 2012; Saeki et al, 2001) that can accommodate our CRISPR/ Cas9 construct (Figure 4a). We first validated this strategy in N2A cells. We found HSV-Cas9-Fto decreased both Fto $\left(\mathrm{F}_{1,4}=28.97, p<0.05\right.$, Supplementary Fig. S2A $)$ and FTO $\left(\mathrm{F}_{1,10}=70.2, p<0.001\right.$, Supplementary Fig. S2C $)$ levels but increased $\mathrm{m} 6 \mathrm{~A}$ levels $\left(\mathrm{F}_{1,4}=8.08, p<0.05\right.$, Supplementary Fig. S2B) compared to HSV-Cas9-Control. Furthermore, microinjection of HSV-Cas9-Fto into dorsal CA1 of mice decreased Fto levels in hippocampus $\left(\mathrm{F}_{1,6}=8.95, p<0.05\right.$, Supplementary Fig. S2D) and decreased FTO protein levels in infected neurons (Figure 4b). Together these findings provide both in vitro and in vivo validation of FTO depletion in the dorsal hippocampus using this technique. 
a
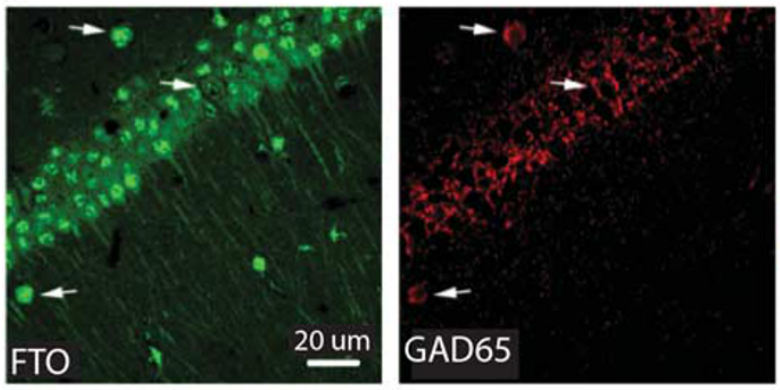

b
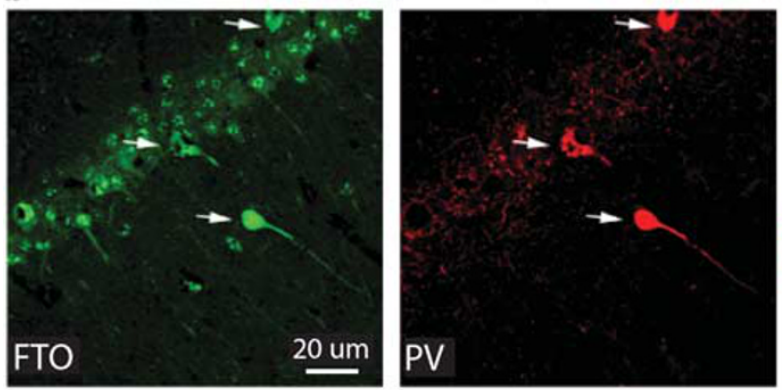

C
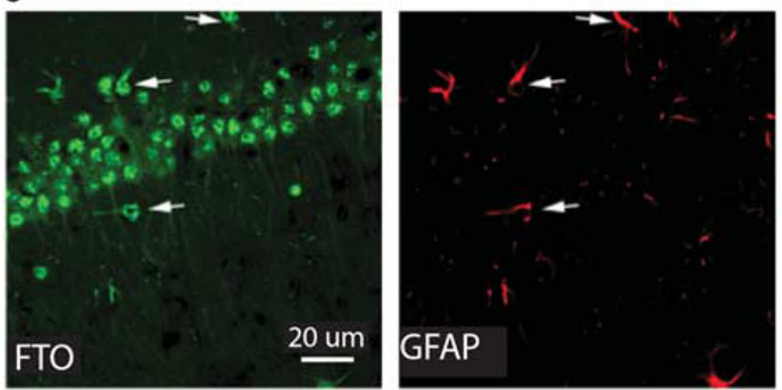
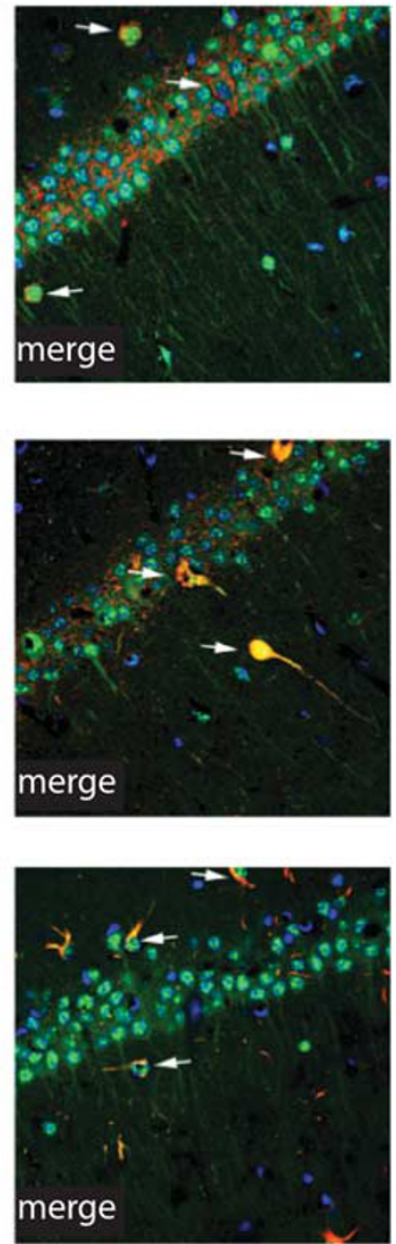

Figure I Cellular and subcellular distribution of FTO in dorsal hippocampus. (a-c) FTO (green) is robustly expressed in CAI neurons in the dorsal hippocampus. In neurons, this expression is robust in the cell body, but also observed in dendrites. (a) FTO is also expressed in glial cells and interneurons, as evidenced by co-expression of FTO with cells expressing the GABA cell marker GAD65 (red), (b) the interneuronal marker parvalbumin (PV, red), or (c) the glial marker GFAP (red). The merged image contains the DNA stain DAPI (blue). Arrows indicate the same cell across images.

We used this novel strategy to examine the effects of decreasing FTO levels in the dorsal hippocampus on memory formation. We microinjected HSV-Cas9-Fto into the CA1 region of dorsal hippocampus 4 days before contextual fear conditioning and found memory measured $24 \mathrm{~h}$ after training was enhanced compared to mice injected with HSV-Cas9-Control $\left(\mathrm{F}_{1,12}=6.96 ; p<0.05\right.$, Figure $\left.4 \mathrm{c}\right)$.

To further confirm the effects of the novel HSV-Cas9-Fto virus, we designed an HSV expressing shRNA against Fto (Figure 4d). Similar to HSV-Cas9-Fto, HSV-shRNA-Fto decreased both Fto $\left(\mathrm{F}_{1,4}=88.90, p<0.001\right.$, Supplementary Fig. S3A $)$ and FTO $\left(\mathrm{F}_{1,10}=9.31 p<0.05\right.$, Supplementary Fig. $\mathrm{S} 3 \mathrm{C})$ levels but increased m6A levels $\left(\mathrm{F}_{1,4}=35.56, p<0.05\right.$, Supplementary Fig. S3B) in N2A cells. HSV-shRNA-Fto microinjection into the dorsal CA1 region of the hippocampus (Figure 4e) also decreased hippocampal expression of Fto $\left(\mathrm{F}_{1,12}=7.90, p<0.05\right.$, Supplementary Fig. S3D) and decreased FTO levels in infected neurons (Figure 4e). Again, similar to HSV-Cas9-Fto, HSV-shRNA-Fto microinjected into CA1 region of dorsal hippocampus $4 \mathrm{~d}$ before contextual fear conditioning enhanced subsequent memory expression $\left(\mathrm{F}_{1,12}=8.35, p<0.05\right.$, Figure $\left.4 \mathrm{f}\right)$. Therefore, both CRISPR/
Cas9 and shRNA targeting of FTO in the dorsal hippocampus of mice produced a similar behavioral profile. Neither HSV-CRISPR/Cas9-Fto nor HSV-shRNA-Fto affected reactivity to the foot shock during training (Cas9: $\mathrm{F}_{1,9}=0.41$, $p>0.05$ Supplementary Fig. S2E; shRNA $F_{1,10}=0.09$, $p>0.05$, Supplementary Fig. S3E) or post-shock freezing during training (post-shock freezing, Cas9: $\mathrm{F}_{1,14}=0.07$, $p>0.05$, Supplementary Fig. S2F; shRNA: $F_{1,11}=0.15$, $p>0.05$, Supplementary Fig. S3F), indicating that the effects of FTO depletion are specific to memory formation.

\section{DISCUSSION}

The present data provide novel evidence that FTO and mRNA methylation are actively regulated in the dorsal hippocampus in response to learning. First, we showed that the RNA demethylase FTO is present near synapses. Second, we found that levels of Fto decrease, whereas levels of methylated mRNA increase, in response to fear conditioning. Fto depletion was not observed in the context only or shock only control training groups, suggesting that particularly salient events are necessary to trigger FTO removal. Third, 
a
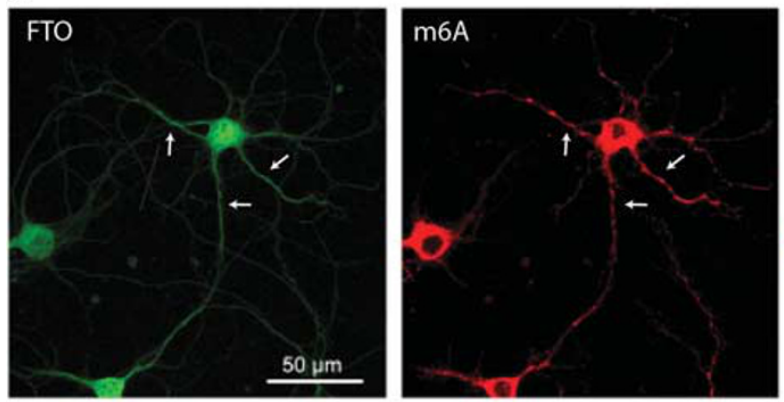

b
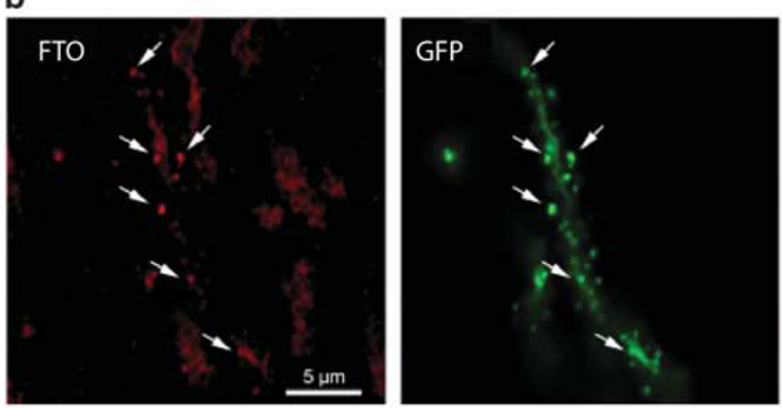

C

FTO

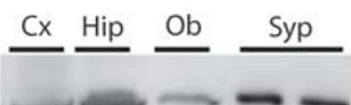

$\mathrm{N}$-Cadherin

GAPDH
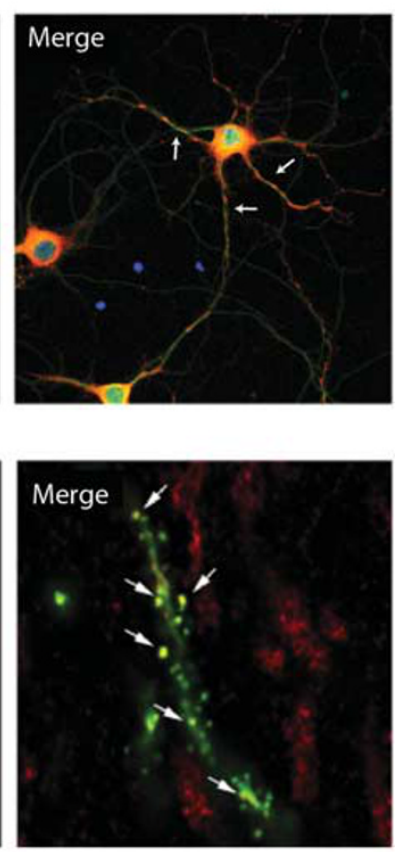

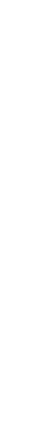

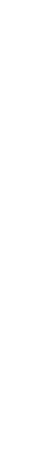


a

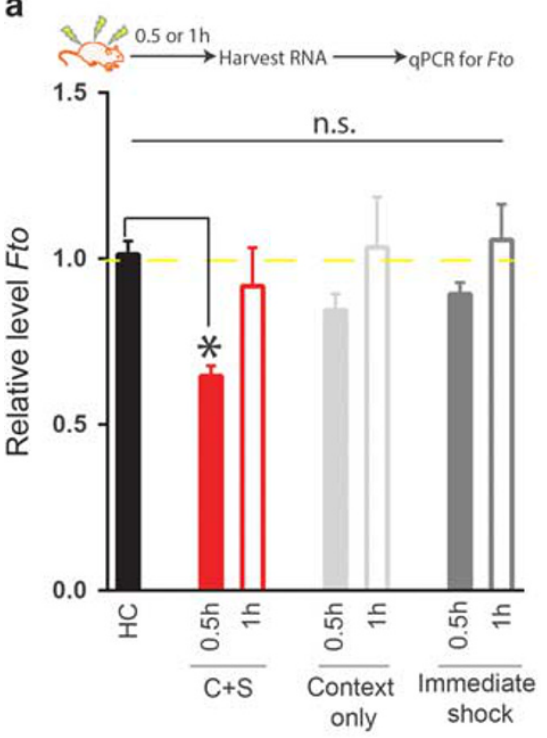

b

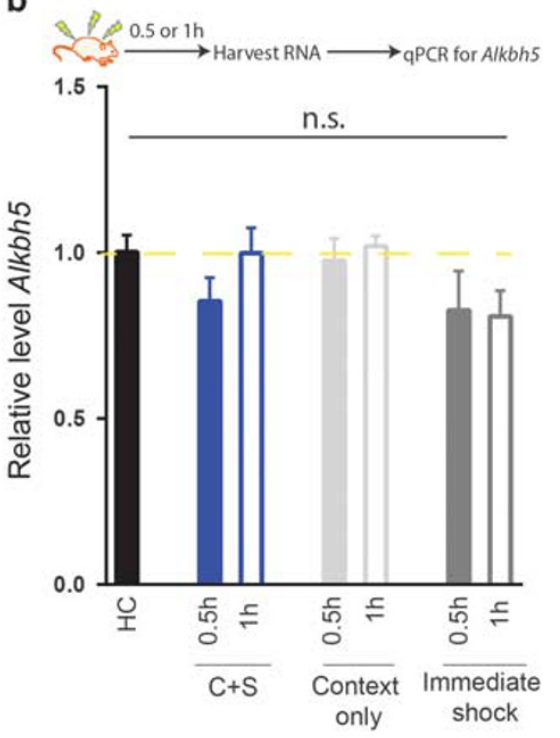

C
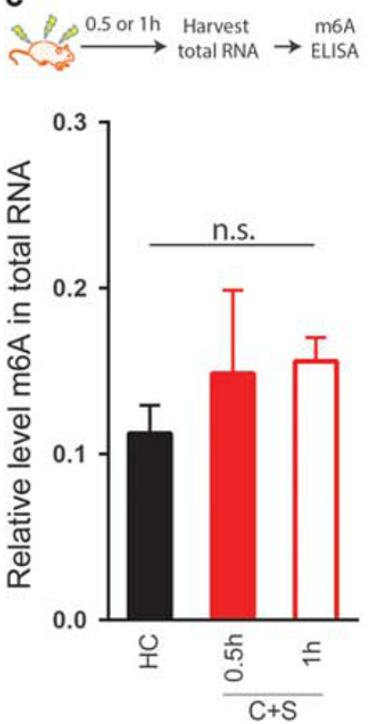

d
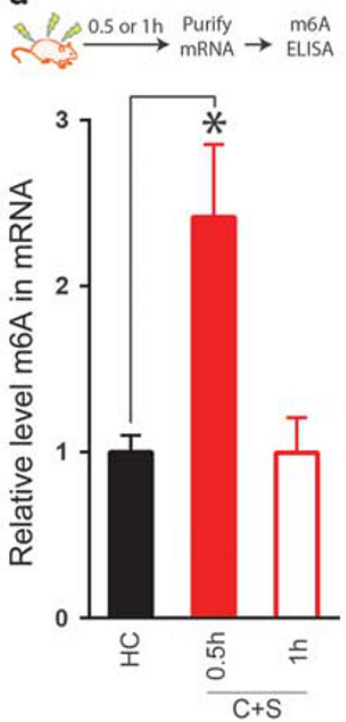

e
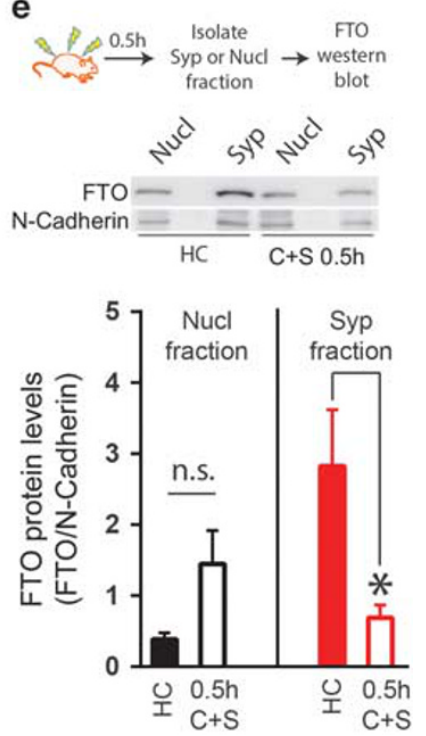

Figure 3 Contextual fear conditioning alters expression of Fto and methylated mRNA in dorsal hippocampus. (a) Mice were contextually fear conditioned $(\mathrm{C}+\mathrm{S})$ and $\mathrm{CA}$ I region of dorsal hippocampus harvested either 0.5 or I h later. Control groups received context placement alone (Context alone), immediate shock alone (Immediate shock) or were taken directly from the homecage (HC). Fto expression was decreased $0.5 \mathrm{~h}$ after fear conditioning $(\mathrm{C}+\mathrm{S}) \mathrm{compared}$ to all other groups, which did not differ from one another (HC $(n=16), C+S 0.5 \mathrm{~h}(n=10)$, all other conditions $(n=8))$. (b) In contrast, fear conditioning did not affect Alkbh5 expression (HC ( $n=13), C+S 0.5 \mathrm{~h}(n=10)$, Context only $(n=8), C+S \mid h(n=8), n=5$ for all other conditions). (c) Fear conditioning had no effect on m6A levels in total RNA (HC $(n=7), n=4$ for all other conditions), (d) but m6A levels of mRNA were increased $0.5 \mathrm{~h}$ after fear conditioning ( $\mathrm{HC}(n=7), n=4$ for all other conditions). (e) Fear conditioning decreased FTO in the synaptoneurosomal (Syp) fraction $0.5 \mathrm{~h}$ after training $(\mathrm{HC}(n=3)$, $\mathrm{C}+\mathrm{S}$ $0.5 \mathrm{~h}(n=4))$, with no effect on the nuclear (Nucl) abundance of FTO $(\mathrm{HC}(n=3), \mathrm{C}+\mathrm{S}(n=4))$. Dashed yellow line represents HC control. Mean \pm S.E.M., $* p<0.05$.

anti-memory transcripts by demethylating the transcripts at different loci within mRNA. The observation that expression of Alkbh5, the other known RNA demethylase, does not change in expression after fear conditioning suggests that different RNA demethylases, and therefore their targets, are differentially controlled by memory formation. This suggests that RNA methylation exerts different influences on different transcripts after memory formation.

Given the limits of current knockdown technology, our FTO depletion necessarily targeted total FTO levels across all cellular fractions, making it unclear if the synaptic and/or nuclear function of FTO underlies the observed memory enhancement. Teasing apart individual genes or sites that FTO targets in the synaptic versus nuclear compartments will be vital to unlocking the specific function of mRNA methylation in memory formation.

Our findings are consistent with a recent study showing that virally delivered shRNA targeting FTO in the medial prefrontal cortex enhances expression of auditory cue fear memory (Widagdo et $a l, 2016$ ). We show that a similar process occurs in the hippocampus, suggesting that the regulation of mRNA methylation may be a universal mechanism in memory formation. In addition, our novel observation that synaptic FTO is preferentially decreased by 
a

\begin{tabular}{|l|l|}
\hline Synapsin-Cas9-2A-GFP & U6-Fto-sgRNA \\
\hline
\end{tabular}

b
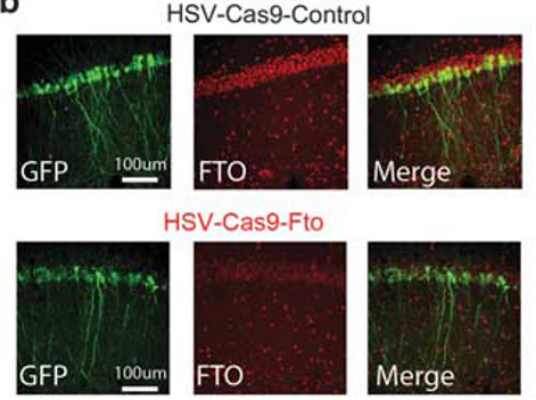

HSV-Cas9-Fto
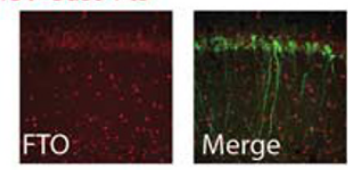

c

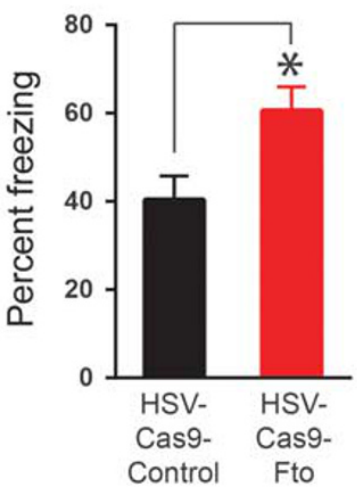

d

HSV-shRNA-Fto

\begin{tabular}{|l|l|}
\hline CMV-GFP & U6-Fto-shRNA \\
\hline
\end{tabular}

e
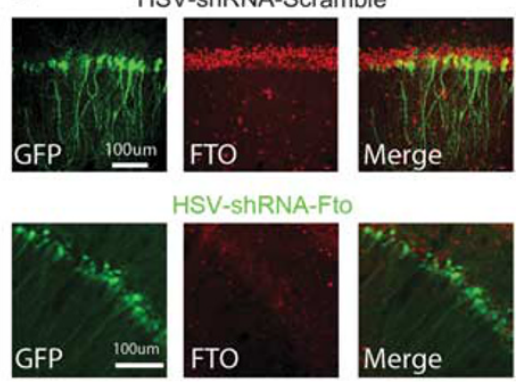

f

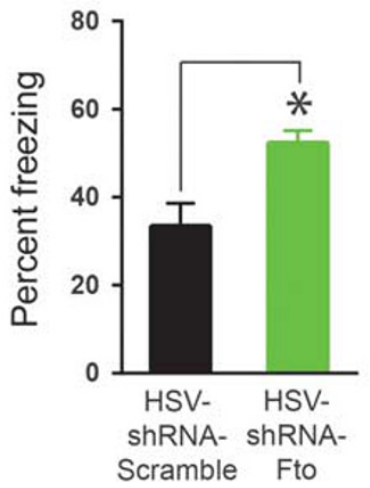

Figure 4 Artificially decreasing FTO in dorsal hippocampus enhances memory. (a) Schematic of HSV-CRISPR/Cas9 construct to target Fto (HSV-Cas9-Fto). (b) HSV-Cas9-Fto microinjected into dorsal CAI region of dorsal hippocampus infected principal (excitatory) neurons where it reduced FTO expression. (c) Microinjecting HSV-Cas9-Fto enhanced memory assessed $24 \mathrm{~h}$ after contextual fear conditioning ( $\mathrm{n}=7$ for both conditions). ( $d$, e) To validate the effects of HSV-Cas9-Fto, an HSV expressing shRNA targeted against Fto (HSV-shRNA-Fto) was also microinjected into CAI region of dorsal hippocampus, and reduced FTO expression in infected neurons. (f) HSV-shRNA-Fto similarly enhanced memory compared to HSV expressing a scrambled control (HSV-shRNA-control $(n=8)$, HSV-shRNA-Fto $(n=6))$. Mean \pm SEM, * $p<0.05$.

learning suggests that mRNA methylation and demethylation may have complex and neuronal compartment-specific functions, necessitating compartment-specific analysis of the targets of FTO and mRNA methylation.

The ability of FTO to constrain hippocampus-dependent memory suggests that FTO may help maintain the sparseness of the hippocampal memory trace, potentially aiding in the prevention of memory generalization. In this way, FTO may actively interfere with neuronal incorporation into an engram under basal conditions. Degradation or removal of FTO from the synapse may facilitate the incorporation of that neuron into the new engram.

The present findings further implicate mRNA methylation as an important novel component in memory formation and further suggest that m6A-binding proteins and local demethylation in synapses may be instrumental to precisely defining the role of mRNA methylation in memory formation.

\section{FUNDING AND DISCLOSURE}

This work was supported by grants from the Canadian Institutes of Health Research (CIHR, grant numbers MOP-74650 to SAJ, FDN143227 to PWF), Natural Science and Engineering Council of Canada (NSERCs to SAJ and
PWF), Brain Canada (SAJ) and a Brain \& Behavior Foundation grant (SAJ). BJW was partially supported by a SickKids Research Training Centre Restracomp Fellowship (to BJW). SAJ and PWF are both members of the Canadian Institute for Advanced Research (CIFAR). The authors declare no conflict of interest.

\section{REFERENCES}

Anagnostaras SG, Josselyn SA, Frankland PW, Silva AJ (2000). Computer-assisted behavioral assessment of Pavlovian fear conditioning in mice. Learn Mem 7: 58-72.

Blanchard RJ, Blanchard DC (1969). Passive and active reactions to fear-eliciting stimuli. J Compar Physiol Psychol 68: 129-135.

Bolles RC, Fanselow MS (1982). Endorphins and behavior. Annu. Rev. Psychol. 33: 87-101.

Branson K, Robie AA, Bender J, Perona P, Dickinson MH (2009). High-throughput ethomics in large groups of Drosophila. Nat Methods 6: 451-457.

Cole CJ, Mercaldo V, Restivo L, Yiu AP, Sekeres MJ, Han JH et al (2012). MEF2 negatively regulates learning-induced structural plasticity and memory formation. Nat Neurosci 15: 1255-1264.

Davis HP, Squire LR (1984). Protein synthesis and memory: a review. Psychol Bull 96: 518-559.

Dominissini D, Moshitch-Moshkovitz S, Schwartz S, Salmon-Divon M, Ungar L, Osenberg S et al (2012). Topology of the human and 
mouse m6A RNA methylomes revealed by m6A-seq. Nature 485 : 201-206.

Duvarci S, Nader K, LeDoux JE (2008). De novo mRNA synthesis is required for both consolidation and reconsolidation of fear memories in the amygdala. Learn Mem 15: 747-755.

Fanselow M (1990). Factors governing one-trial contextual conditioning. Anim Learn Behav 18: 264-270.

Frankland PW, Cestari V, Filipkowski RK, McDonald RJ, Silva AJ (1998). The dorsal hippocampus is essential for context discrimination but not for contextual conditioning. Behav Neurosci 112: 863-874.

Frankland PW, Josselyn SA, Anagnostaras SG, Kogan JH, Takahashi E, Silva AJ (2004). Consolidation of CS and US representations in associative fear conditioning. Hippocampus 14: 557-569.

Fu Y, Dominissini D, Rechavi G, He C (2014). Gene expression regulation mediated through reversible $\mathrm{m}(6) \mathrm{A}$ RNA methylation. Nat Rev Genet 15: 293-306.

Han JH, Kushner SA, Yiu AP, Hsiang HL, Buch T, Waisman A et al (2009). Selective erasure of a fear memory. Science 323: 1492-1496.

Hernandez PJ, Abel T (2008). The role of protein synthesis in memory consolidation: progress amid decades of debate. Neurobiol Learn Mem 89: 293-311.

Hess ME, Hess S, Meyer KD, Verhagen LA, Koch L, Bronneke HS et al (2013). The fat mass and obesity associated gene (Fto) regulates activity of the dopaminergic midbrain circuitry. Nat Neurosci 16: 1042-1048.

Hoernes TP, Clementi N, Faserl K, Glasner H, Breuker K, Lindner $\mathrm{H}$ et al (2015). Nucleotide modifications within bacterial messenger RNAs regulate their translation and are able to rewire the genetic code. Nucleic Acids Res 44: 852-862.

Ji J, Maren S (2008). Differential roles for hippocampal areas CA1 and $\mathrm{CA} 3$ in the contextual encoding and retrieval of extinguished fear. Learn Mem 15: 244-251.

Jia G, Fu Y, He C (2013). Reversible RNA adenosine methylation in biological regulation. Trends Genet 29: 108-115.

Linder B, Grozhik AV, Olarerin-George AO, Meydan C, Mason CE, Jaffrey SR (2015). Single-nucleotide-resolution mapping of m6A and m6Am throughout the transcriptome. Nat Methods 12: 767-772.

Liu N, Pan T (2016). N6-methyladenosine-encoded epitranscriptomics. Nat Struct Mol Biol 23: 98-102.

McTaggart JS, Lee S, Iberl M, Church C, Cox RD, Ashcroft FM (2011). FTO is expressed in neurones throughout the brain and its expression is unaltered by fasting. PLoS ONE 6: e27968.

Meyer KD, Saletore Y, Zumbo P, Elemento O, Mason CE, Jaffrey SR (2012). Comprehensive analysis of mRNA methylation reveals enrichment in 3' UTRs and near stop codons. Cell 149: 1635-1646.

Nakazawa Y, Pevzner A, Tanaka KZ, Wiltgen BJ (2016). Memory retrieval along the proximodistal axis of CA1. Hippocampus 26: $1140-1148$.

Neve RL (2012). Overview of gene delivery into cells using HSV-1based vectors. Curr Protoc Neurosci Chapter 4: Unit 412.

Niu Y, Zhao X, Wu YS, Li MM, Wang XJ, Yang YG (2013). N6-methyl-adenosine (m6A) in RNA: an old modification with a novel epigenetic function. Genomics Proteomics Bioinformatics 11: 8-17.

Paxinos G, Franklin KBJ (2004). The Mouse Brain in Stereotaxic Coordinates, Compact, 2nd edn. Elsevier Academic Press: Amsterdam; Boston.
Pilo Boyl P, Di Nardo A, Mulle C, Sassoe-Pognetto M, Panzanelli P, Mele A et al (2007). Profilin2 contributes to synaptic vesicle exocytosis, neuronal excitability, and novelty-seeking behavior. EMBO J 26: 2991-3002.

Saeki Y, Fraefel C, Ichikawa T, Breakefield XO, Chiocca EA (2001). Improved helper virus-free packaging system for HSV amplicon vectors using an ICP27-deleted, oversized HSV-1 DNA in a bacterial artificial chromosome. Mol Ther 3: 591-601.

Sanders MJ, Wiltgen BJ, Fanselow MS (2003). The place of the hippocampus in fear conditioning. Eur J Pharmacol 463: 217-223.

Sarbassov DD, Guertin DA, Ali SM, Sabatini DM (2005). Phosphorylation and regulation of $\mathrm{Akt} / \mathrm{PKB}$ by the rictormTOR complex. Science 307: 1098-1101.

Schwartz S, Mumbach MR, Jovanovic M, Wang T, Maciag K, Bushkin GG et al (2014). Perturbation of m6A writers reveals two distinct classes of mRNA methylation at internal and 5' sites. Cell Rep 8: 284-296.

Sekeres MJ, Neve RL, Frankland PW, Josselyn SA (2010). Dorsal hippocampal CREB is both necessary and sufficient for spatial memory. Learn Mem 17: 280-283.

Steiner I, Kennedy PG, Pachner AR (2007). The neurotropic herpes viruses: herpes simplex and varicella-zoster. Lancet Neurol 6: $1015-1028$.

Sweatt JD (2013). The emerging field of neuroepigenetics. Neuron 80: 624-632.

Tanaka KZ, Pevzner A, Hamidi AB, Nakazawa Y, Graham J, Wiltgen BJ (2014). Cortical representations are reinstated by the hippocampus during memory retrieval. Neuron 84: 347-354.

Wang X, Zhao BS, Roundtree IA, Lu Z, Han D, Ma H et al (2015). $\mathrm{N}(6)$-methyladenosine modulates messenger RNA translation efficiency. Cell 161: 1388-1399.

Wang Y, Li Y, Toth JI, Petroski MD, Zhang Z, Zhao JC (2014). N6-methyladenosine modification destabilizes developmental regulators in embryonic stem cells. Nat Cell Biol 16: 191-198.

Widagdo J, Zhao Q-Y, Kempen M-J, Tan MC, Ratnu VS, Wei W et al (2016). Experience-dependent accumulation of N6-methyladenosine in the prefrontal cortex is associated with memory processes in mice. J Neurosci 36: 6771-6777.

Wiltgen BJ, Sanders MJ, Anagnostaras SG, Sage JR, Fanselow MS (2006). Context fear learning in the absence of the hippocampus. J Neurosci 26: 5484-5491.

Wiltgen BJ, Sanders MJ, Behne NS, Fanselow MS (2001). Sex differences, context preexposure, and the immediate shock deficit in Pavlovian context conditioning with mice. Behav Neurosci 115: 26-32.

Wiltgen BJ, Zhou M, Cai Y, Balaji J, Karlsson MG, Parivash SN et al (2010). The hippocampus plays a selective role in the retrieval of detailed contextual memories. Curr Biol 20: 1336-1344.

Wood SC, Anagnostaras SG (2011). Interdependence of measures in pavlovian conditioned freezing. Neurosci Lett 505: 134-139.

Zhao X, Yang Y, Sun BF, Shi Y, Yang X, Xiao W et al (2014). FTO-dependent demethylation of N6-methyladenosine regulates mRNA splicing and is required for adipogenesis. Cell Res 24: 1403-1419.

Zhou J, Wan J, Gao X, Zhang X, Jaffrey SR, Qian SB (2015). Dynamic m(6)A mRNA methylation directs translational control of heat shock response. Nature 526: 591-594.

Zovkic IB, Paulukaitis BS, Day JJ, Etikala DM, Sweatt JD (2014). Histone H2A.Z subunit exchange controls consolidation of recent and remote memory. Nature 515: 582-586.

Supplementary Information accompanies the paper on the Neuropsychopharmacology website (http://www.nature.com/npp) 